\title{
Walking performance in people with diabetic neuropathy: benefits and threats
}

\author{
R. V. Kanade • R. W. M. van Deursen • K. Harding • \\ P. Price
}

Received: 2 November 2005 / Accepted: 31 March 2006 / Published online: 7 June 2006

(C) Springer-Verlag 2006

\begin{abstract}
Aims/hypothesis Walking is recommended as an adjunct therapy to diet and medication in diabetic patients, with the aim of improving physical fitness, glycaemic control and body weight reduction. Therefore we evaluated walking activity on the basis of capacity, performance and potential risk of plantar injury in the diabetic population before it can be prescribed safely.

Subjects, materials and methods Twenty-three subjects with diabetic neuropathy (DMPN) were compared with 23 patients with current diabetic foot ulcers, 16 patients with partial foot amputations and 22 patients with trans-tibial amputations. The capacity for walking was measured using a total heart beat index (THBI). Gait velocity and average daily strides were measured to assess the performance of walking, and its impact on weight-bearing was studied using maximum peak pressure.

Results THBI increased $(p<0.01)$ and gait velocity and daily stride count fell ( $p<0.001$ for both) with progression of foot complications. The maximum peak pressures over the affected foot of patients with diabetic foot ulcers $(p<0.05)$ and partial foot amputations $(p<0.01)$ were higher than in the group with DMPN. On the contralateral side, the diabetic foot ulcer group showed higher maximum peak
\end{abstract}

R. V. Kanade $\cdot$ R. W. van Deursen $(\bowtie)$

Research Centre for Clinical Kinaesiology,

Department of Physiotherapy, Cardiff University,

Ty Dewi Sant, Heath Park,

Cardiff CF14 4XN, UK

e-mail: vandeursenr@cardiff.ac.uk

K. Harding $\cdot$ P. Price

Wound Healing Research Unit, School of Medicine,

Cardiff University,

Cardiff, UK pressure over the total foot $(p<0.05)$, and patients with partial foot amputations $(p<0.01)$ and trans-tibial amputations $(p<0.05)$ showed higher maximum peak pressure over the heel.

Conclusions/interpretation Walking capacity and performance decrease with progression of foot complications. Although walking is recommended to improve fitness, it cannot be prescribed in isolation, considering the increased risk of plantar injury. For essential walking we therefore recommend the use of protective footwear. Walking exercise should be supplemented by partial or non-weightbearing exercises to improve physical fitness in diabetic populations.

Keywords Diabetic foot ulcers · Diabetic neuropathy . Partial foot amputations · Peak pressures · Physical fitness . Trans-tibial amputation · Walking

\begin{tabular}{ll}
\multicolumn{2}{l}{ Abbreviations } \\
DFU & diabetic foot ulcer \\
DMPN & diabetic neuropathy \\
H-R QOL & health-related quality of life \\
LEA & lower extremity amputation \\
MPP & maximum peak pressure \\
MT & metatarsal \\
PFA & partial foot amputation \\
SAM & step activity monitor \\
THBI & total heart beat index \\
TTA & trans-tibial amputation
\end{tabular}

\section{Introduction}

Walking is a common activity of daily life. It is recommended as an adjunct therapy to dietary treatment in obese 
type 2 diabetic patients, not only for body weight reduction, but also for improvement of insulin sensitivity [1]. Ten thousand steps per day are reported to result in improved glucose tolerance and a reduction in systolic and diastolic blood pressure in overweight women at risk of type 2 diabetes [2]. Another study recommends brisk walking as a preferred mode of aerobic exercise for a minimum of 30 min on most days of the week [3].

Although the positive effects of walking activity on the cardio-respiratory system and glycaemic control are established, concerns should be raised with respect to the effect on the feet, since there is a serious risk of foot complications in the presence of diabetic neuropathy (DMPN). The estimated prevalence of DMPN is nearly $50 \%$ among the diabetic population [4]. Diabetic foot complications usually follow a trend following peripheral neuropathy, beginning with ulceration [4] causing partial foot amputation (PFA) and ultimately resulting in major lower extremity amputation (LEA). Foot ulcers precede the vast majority (85\%) of LEAs in patients with diabetes mellitus [5]. Soft tissue and bone infections that do not respond to vigorous local care and i.v. antibiotic therapy may require amputation [6]. In an attempt to salvage the foot for ambulation, PFA is often performed [7]. PFAs often predispose the diabetic patient to increased foot pressures and development of foot deformities, which further increase their risk of ulceration and amputation [8].

Walking activity has been quantified in some of these groups of patients with foot complications [9, 10]. Also, there is substantial literature available discussing the plantar pressure distribution in this patient population [11-13]. However, functional outcome based on measures of capacity and performance [14], along with the impact on weight-bearing, needs further investigation in the diabetic population with foot complications. Not enough is known about the advantages and disadvantages of walking, making it difficult to provide appropriate recommendations for exercise in this patient population.

Therefore we aimed to study the capacity and performance in relation to walking activity and its impact on the plantar tissues across the various groups with DMPN at sequential stages of foot complications, namely (1) DMPN with no history of further complications; (2) DMPN with unilateral current diabetic foot ulcers (DFUs) on the plantar surface; (3) DMPN with unilateral healed PFAs; and (4) DMPN with unilateral trans-tibial amputation (TTA). We predicted that the capacity and performance of walking would decrease and the potential risk of plantar injury based on peak plantar pressures would increase with the progression of foot complications.

\section{Subjects, materials and methods}

Ethical approval

The study was approved by the Cardiff and Vale NHS Trust Research \& Development Office and the South East Wales Local Research Ethics Committee, UK. The investigations were carried out in accordance with the principles of the Declaration of Helsinki as revised in 2000.

\section{Subjects}

Following written informed consent, 23 control subjects with no history of plantar ulceration, 23 subjects with unilateral current plantar ulceration (heel ulcers $=5$; first metatarsal [MT] head=7; second MT head=1; fifth MT head $=4$; hallux $=4$; second toe $=1$; fourth toe $=1$ ), 16 subjects with healed unilateral PFAs (trans-MT=5; ray=4; hallux $=5$; all five toes $=1$; first two=1), and 22 subjects with healed unilateral TTA participated in this cross-sectional study.

Patients with DFUs, PFAs and TTAs were matched on marginal distributions with respect to weight, height and BMI with the 23 control subjects. All 84 subjects were known cases of DMPN. Amputations of the ray or hallux with or without toes and trans-MT amputations were classified as PFAs for this study.

Semmes-Weinstein monofilaments were used to confirm the neuropathy status [15]. Neuropathy was considered present if the 5.07 (10 g) Semmes-Weinstein monofilament (loss of protective sensation) was not perceived in at least one of the four plantar areas tested (heel, first MT head, fifth MT head and hallux). Between five to ten trials were performed at each site [16] and the subject needed to perceive $80 \%$ of the trials to be graded as the sensation intact over that site. The site was scored 1 in the case of the presence of sensation and 0 in the case of absence of the sensation. The sum of the scores over the four sites was used to present the final sensory score over the entire foot (Table 1).

Selection criteria

Subjects within the age range of 40 to 75 years, living independently in the community, with visual acuity of a minimum of 20/40 in the better eye (pre-requisite to obtain a driving licence) and an ability to communicate without support were included. All the participants were capable of walking independently to perform their activities of daily life with or without a walking aid. However, no walking aids were used during the testing procedures. The patients with TTAs always used their artificial limb for walking. All the subjects with TTAs had completed at least 6 months following rehabilitation at the time of discharge 
Table 1 Characteristics of the patients from all four groups

\begin{tabular}{|c|c|c|c|c|}
\hline Subject characteristics & DMPN group $(n=23)$ & DFU group $(n=23)$ & PFA group $(n=16)$ & TTA group $(n=22)$ \\
\hline Age $($ mean $\pm \mathrm{SD})$ & $64.48 \pm 5.75$ & $59.74 \pm 9.55$ & $62.13 \pm 8.83$ & $62.86 \pm 6.08$ \\
\hline $\operatorname{Sex}(F / M)$ & $3 / 20$ & $4 / 19$ & $1 / 15$ & $2 / 20$ \\
\hline Type of diabetes $(1 / 2)$ & $12 / 11$ & $3 / 20$ & $7 / 9$ & $6 / 16$ \\
\hline Height, cm (mean \pm SD) & $175.47 \pm 7.98$ & $176.90 \pm 9.04$ & $175.54 \pm 8.73$ & $174.14 \pm 5.34$ \\
\hline Mass, $\mathrm{kg}($ mean \pm SD $)$ & $95.51 \pm 9.82$ & $98.48 \pm 19.54$ & $93.98 \pm 18.90$ & $95.45 \pm 14.65$ \\
\hline BMI, $\mathrm{kg} / \mathrm{m}^{2}($ mean $\pm \mathrm{SD})$ & $31.11 \pm 3.37$ & $31.47 \pm 6.19$ & $30.5 \pm 6.12$ & $31.42 \pm 4.21$ \\
\hline \multicolumn{5}{|c|}{ Sensory score, $\min =0 ; \max =4$ (no. of subjects) } \\
\hline 0 & 4 & 19 & 11 & 14 \\
\hline 1 & 5 & 3 & 3 & 1 \\
\hline 2 & 12 & 1 & 1 & 1 \\
\hline 3 & 2 & & 1 & 6 \\
\hline
\end{tabular}

Groups were matched on marginal distributions for height, weight and BMI. The plantar sensory score indicates that the patients with further foot complications such as DFUs, PFAs and TTAs were more neuropathic compared with the group with DMPN

from Artificial Limb and Appliance Centres (South Wales).

Exclusion criteria

Patients with bilateral LEAs or any major neurological and musculo-skeletal impairment other than those resulting from diabetic foot complications, for example, diabetic peripheral neuropathy or painful forms of DMPN, DFUs, PFAs or TTAs were excluded from the study. Subjects taking medication with known effects on the central nervous system, or with known dependence on alcohol and/or drugs were excluded. Patients presenting with acute symptoms of nephropathy or cardiovascular complications resulting from diabetes mellitus or any other major chronic illnesses were excluded as well.

Instrumentation and procedure

The capacity for walking was measured using the total heart beat index (THBI), which was calculated as an index of energy expenditure [17] during a 2-min walk test [18]. The patients walked at their natural self-selected speed. A heart rate monitor (Polar S8 10i; Polar Electro OY, Kempele, Finland) was used to record the total number of heart beats, and the total distance covered during the walk test was recorded. THBI was computed as the ratio of the total number of heart beats to the total distance covered [17].

Step activity monitors (SAMs) (Prosthetics Research Study, Seattle, WA, USA) were used to record the performance of daily walking activity [19]. The SAM is a reliable and valid tool for the measurement and recording of walking activity [20]. The accelerometer-based device measuring $680 \times 50 \times 20 \mathrm{~mm}$ was strapped around the subject's right leg laterally above the ankle joint and the parameters were optimised according to the height and gait characteristics of each individual subject. Subjects were instructed to wear the SAM continuously for eight consecutive days, except when bathing, showering or swimming. The number of strides every 1-min interval were recorded for a continuous $24 \mathrm{~h}$ over seven consecutive days. The mean average daily stride count was considered for analysis (one stride equals two [right and left] steps).

Gait velocity was chosen as a measure of gait performance and measured using a digital video camera at a sampling rate of $25 \mathrm{~Hz}$. Each subject walked a distance of $12 \mathrm{~m}$ at their self-selected pace. Three trials were recorded for each subject [21] as they walked on level ground between two parallel calibration sticks placed $1 \mathrm{~m}$ apart.

The impact of walking activity on weight-bearing was studied based on the plantar pressure distribution. Maximum peak pressure (MPP) was measured during the gait measurement using the Pedar in-shoe pressure measurement system (Novel, Munich, Germany) at a sampling rate of $50 \mathrm{~Hz}$. MPP reflects the absolute peak pressure during the gait cycle. All patients were measured in uniform standard footwear with appropriate shoe fillers for the patients with PFAs. Two-millimetre thick insoles available in different standard and wide sizes were calibrated using the standard calibration device with homogeneous air pressure ranging from 4 to $60 \mathrm{~N} / \mathrm{cm}^{2}$. Zero measurement was performed before starting the experimental measurement.

Plantar pressure data were analysed using Novel standard software version 10.33. Three trials were recorded for each subject and five mid-gait steps on the right and left foot were selected for analysis from each trial. Individual masks were created for each subject subdividing the foot into six regions, namely: heel, midfoot, medial MTs (first and second MTs), lateral MTs (third, fourth and fifth MTs), hallux, and all the toes. The plantar pressure data obtained from the affected foot of the patients with DFUs and PFAs were compared with data from the right foot of the control 
Table 2 Comparison of gait velocity, daily walking activity and THBI (means \pm SD) of the subjects across the four groups

\begin{tabular}{lccccc}
\hline Walking activity & DMPN group & DFU group & PFA group & TTA group & $p$ value for linear polynomial contrast \\
\hline Gait velocity (m/s) & $1.1 \pm 0.2$ & $0.9 \pm 0.3$ & $0.9 \pm 0.2$ & $0.7 \pm 0.2$ & $<0.001^{* *}$ \\
Daily strides (strides/day) & $4,409 \pm 1,953$ & $2,742 \pm 1,584$ & $3,133 \pm 1,315$ & $1,894 \pm 1.081$ & $<0.001^{* *}$ \\
THBI (beats/min) & $1.2 \pm 0.4$ & $1.6 \pm 0.7$ & $1.9 \pm 0.6$ & $2.0 \pm 1.4$ & $0.019^{*}$ \\
\hline
\end{tabular}

*Significant; ** highly significant

group. Considering the symmetrical pattern of distal polyneuropathy affecting the feet [22] data from the right feet were considered for comparative analysis by default. We confirmed that there was no significant difference in the total MPP between the right and left foot in the control group $(p=0.134)$.

\section{Statistical analysis}

The minimum sample size was calculated to be 23 subjects. Based on a number of gait parameters reported by Mueller et al. [23] a range was found for the standardised difference of between 1.15 and 1.57. For this power calculation a conservative estimate of the standard difference of 1 was used. A power of 0.8 and a significance level of 0.05 were used for this calculation.

No major departures from the necessary parametric assumptions were evident. Linear polynomial contrast within an ANOVA (SPSS 11) was used to investigate the trend of the gait parameters across the four groups. Considering the influence of gait velocity on MPP [24], one-way analysis of covariance was used to compare the means of the MPPs of the adjusted groups with gait velocity as a covariate [25]. Gait velocity was used as a covariate in the analysis of peak plantar pressures because slower walking speed is known to coincide with decreased pressures over some areas of the foot [26]. A significance level of 0.05 was used.

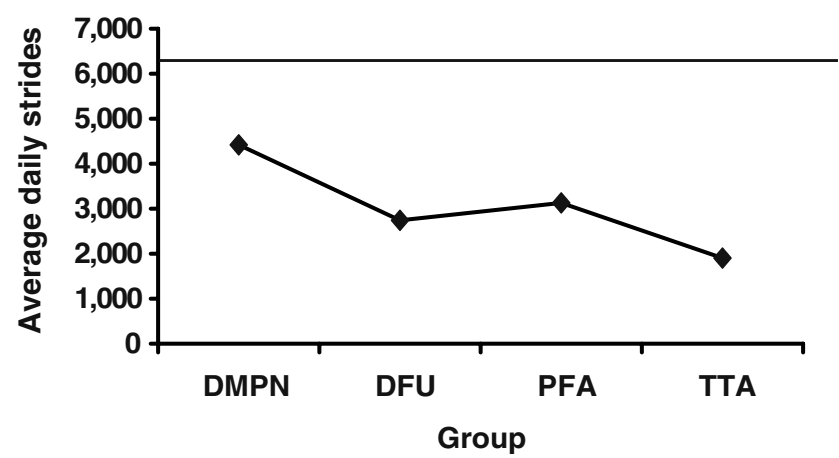

Fig. 1 The average daily stride count decreases from the control diabetic neuropathy (DMPN) group to the group with plantar ulceration (DFU), improves with the partial foot amputation (PFA) group and decreases further with the trans-tibial amputation (TTA) group. The daily walking performance of the diabetic control group is lower compared with the non-diabetic healthy subjects measured by Busse [38]. The horizontal line refers to the data from the reference study

\section{Results}

The subject characteristics of the four groups are presented in Table 1.

THBI, an index of energy expenditure, showed a progressively significant rise across the four groups $(p=0.019)$, as shown in Table 2 , indicating a decline in physical fitness. The average daily strides showed a significant $(p=0.000)$ decline across the groups, demonstrating a decreasing level of activity. However, the graph related to the significant linear polynomial contrast between the groups suggests that the patients with PFAs walked more than the patients with DFUs (Table 2 and Fig. 1). Gait velocity decreased significantly across the four groups from DMPN to TTA $(p=0.000)$.

The descriptive and inferential statistics for the MPP over the affected and the contralateral foot are presented in Table 3. A significant rise in peak pressures was noted over the total plantar area of the foot on the affected limb from DMPN to DFU to PFA group $(p=0.002)$. Regional assessment of specific plantar areas revealed a significant rise in MPP over the midfoot region on the affected limb from DMPN to PFA group ( $p=0.006)$.

On the contralateral limb, the heel $(p=0.015)$ and the medial metatarso-phalangeal region $(p=0.040)$ demonstrated significant difference in peak pressures across the four groups with a significant rise in MPP over the heel $(p=0.005)$.

\section{Discussion}

The present study aimed to investigate the functional outcome of walking activity among the four groups of patients at different stages of foot complications. THBI, average daily stride count, gait velocity and peak pressure were chosen as parameters representative of capacity and performance of walking and its impact on the diabetic foot.

The limitation of this study lies in the choice of a crosssectional design rather than a longitudinal study. The robustness of the study would increase with a longitudinal design to investigate the variations in walking activity as foot complications progress in the diabetic population. However, considering the time-scale required for a longitudinal study and the cost involved we opted to explore our 
Table 3 The descriptive and inferential statistics for the MPP $(\mathrm{kPa}$, means $\pm \mathrm{SD})$ over the affected and the contralateral foot for the four groups are presented along with the post hoc comparisons of the groups with DFUs, PFAs and TTAs with the DMPN group (controls) using gait velocity as the covariate

\begin{tabular}{|c|c|c|c|c|c|c|}
\hline Foot region & DMPN group & DFU group & PFA group & TTA group & $p$ (ANOVA) & $p$ for linear contrast \\
\hline \multicolumn{7}{|l|}{ Total } \\
\hline Affected & $344.5 \pm 82.9$ & $366.9 \pm 72.6$ & $395.7 \pm 110.1$ & $\mathrm{n} / \mathrm{a}$ & $0.006 * *$ & $0.002 * *$ \\
\hline Contralateral & $342.8 \pm 81.3$ & $375.6 \pm 71.1$ & $343.0 \pm 90.0$ & $337.9 \pm 88.7$ & 0.091 & 0.179 \\
\hline \multicolumn{7}{|l|}{ Heel } \\
\hline Affected & $247.5 \pm 41.9$ & $212.7 \pm 77.5$ & $209.5 \pm 59.3$ & $\mathrm{n} / \mathrm{a}$ & 0.674 & 0.461 \\
\hline Contralateral & $247.6 \pm 51.7$ & $239.1 \pm 46.2$ & $270.9 \pm 63.9$ & $246.5 \pm 52.1$ & $0.015^{*}$ & $0.005^{* *}$ \\
\hline \multicolumn{7}{|l|}{ Midfoot } \\
\hline Affected & $88.2 \pm 41.9$ & $80.8 \pm 73.4$ & $194.9 \pm 178.7$ & $\mathrm{n} / \mathrm{a}$ & $0.005^{* *}$ & $0.006^{* *}$ \\
\hline Contralateral & $74.4 \pm 43.2$ & $66.3 \pm 47.1$ & $67.2 \pm 45.0$ & $82.6 \pm 49.4$ & 0.680 & 0.623 \\
\hline \multicolumn{7}{|l|}{ Medial MTs } \\
\hline Affected & $322.1 \pm 93.6$ & $329.6 \pm 123.5$ & $245.9 \pm 167.5$ & $\mathrm{n} / \mathrm{a}$ & 0.167 & 0.565 \\
\hline Contralateral & $322.9 \pm 88.4$ & $367.3 \pm 88.1$ & $307.0 \pm 103.7$ & $306.9 \pm 99.3$ & $0.040^{*}$ & 0.363 \\
\hline \multicolumn{7}{|l|}{ Lateral MTs } \\
\hline Affected & $282.5 \pm 88.5$ & $264.0 \pm 105.6$ & $243.0 \pm 190.7$ & $\mathrm{n} / \mathrm{a}$ & 0.904 & 0.964 \\
\hline Contralateral & $298.1 \pm 78.2$ & $277.7 \pm 72.1$ & $248.6 \pm 54.1$ & $240.4 \pm 84.2$ & 0.738 & 0.454 \\
\hline \multicolumn{7}{|l|}{ Hallux } \\
\hline Affected & $182.2 \pm 81.4$ & $114.2 \pm 116.2$ & $\mathrm{n} / \mathrm{a}$ & $\mathrm{n} / \mathrm{a}$ & 0.100 & 0.100 \\
\hline Contralateral & $177.0 \pm 52.1$ & $157.6 \pm 83.9$ & $146.2 \pm 109.1$ & $165.1 \pm 113.5$ & 0.832 & 0.663 \\
\hline
\end{tabular}

*Significant; **highly significant

Medial MTs First and second metatarsal region, lateral MTs fourth and fifth metatarsal region, n/a not available

research question with a cross-sectional design. However, even a cross-sectional study design posed its own limitations when we were confronted with difficulties in recruiting adequate number of patients with PFAs. Recruitment of adequate numbers of subjects with healed unilateral PFAs was a challenge due to three major factors: (1) the lower incidence of PFAs compared with major LEAs at our centres; (2) the incidence of problems such as wound failure or progression to higher levels of amputation; and

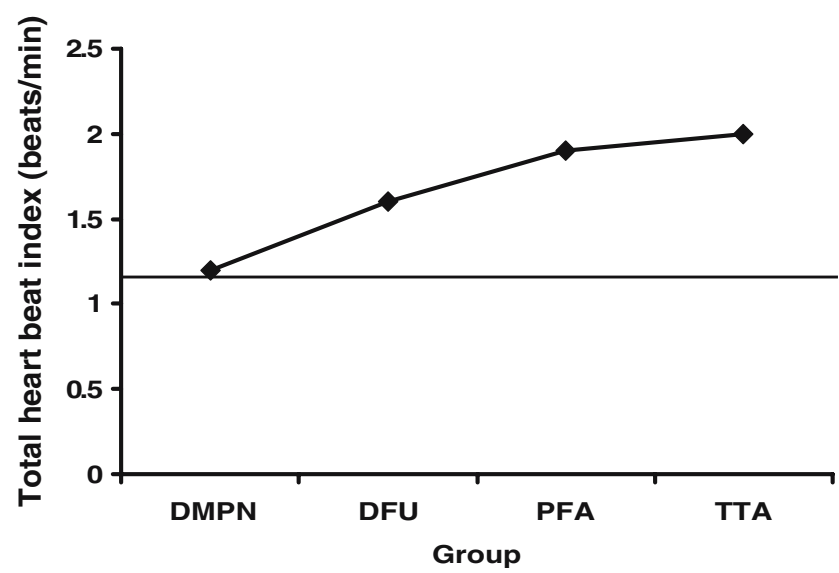

Fig. 2 The gradual rise in the mean total heart beat index (THBI) count across the four diabetic groups indicates an increase in energy expenditure. The THBI score of the control diabetic neuropathy (DMPN) group is comparable with the score of the non-diabetic healthy group presented by Hood et al. [17]. The horizontal line refers to the data from the reference study. DFU, diabetic foot ulcer; PFA, partial foot amputation; TTA, trans-tibial amputation
(3) the presence of acute symptoms of neurological or musculo-skeletal disorders, which might have introduced a confounding bias in the interpretation of the results. However, a reasonable sample size of the PFA group allowed us to compare the four groups statistically.

Our results showed changes in THBI, average daily stride count, gait velocity and peak pressure, demonstrating a decline in the functional capacity and performance of walking and increased risk of plantar injury with progression of foot complications.

Low cardio-respiratory fitness and physical inactivity are independent predictors of all-cause mortality in men with type 2 diabetes mellitus. Therefore it is believed that patients with type 2 diabetes should be encouraged to participate in regular physical activity [27]. Our results confirm the decline in the level of physical fitness demonstrated by increased energy expenditure across the four groups (Fig. 2). Alternatively it could be argued that walking requires more physical exertion (in terms of aerobic capacity) as the level of physical impairment progresses. Therefore the level of physical fitness and the level of physical impairment appear to be interdependent. It is hard to establish a cause-effect relationship between the two factors without a longitudinal study. Irrespectively, the steady rise in the energy expenditure indicates the greater need for improving the level of fitness as the diabetic patients progress from peripheral neuropathy to foot ulceration to minor amputations to major amputations of the lower extremity. 


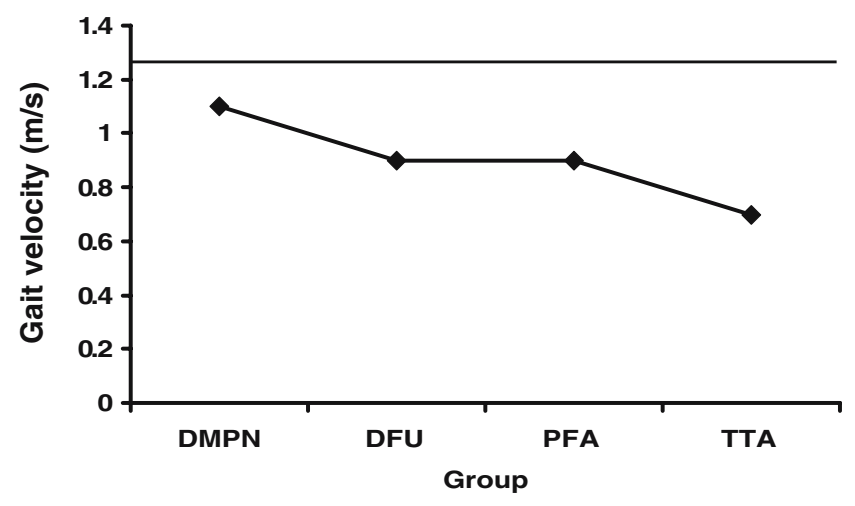

Fig. 3 The gradual decline in the mean values of gait velocity across the four diabetic groups is plotted against the horizontal line referring to the data from the non-diabetic, healthy group measured by Mueller et al. [23]. $D M P N$ Diabetic neuropathy, $D F U$ diabetic foot ulcer, $P F A$ partial foot amputation, TTA trans-tibial amputation

In terms of the performance of walking activity, there was an overall decline in the daily walking performance with progression of foot complications from DMPN to TTA (Fig. 1). However, the graph related to the significant linear polynomial contrast between the groups suggests that the patients with PFAs walked more than the patients with DFUs. The overall decline in walking performance is in tune with the pattern noticed by Tennvall and Apelqvist [28] in the health-related quality of life (H-R QOL) among diabetic people at different stages of foot complications. Diabetic patients with minor amputations are reported to demonstrate better H-R QOL compared with patients with current ulcers, and patients with major amputations are reported to demonstrate lower H-R QOL compared with patients with minor amputations [28]. Although we could

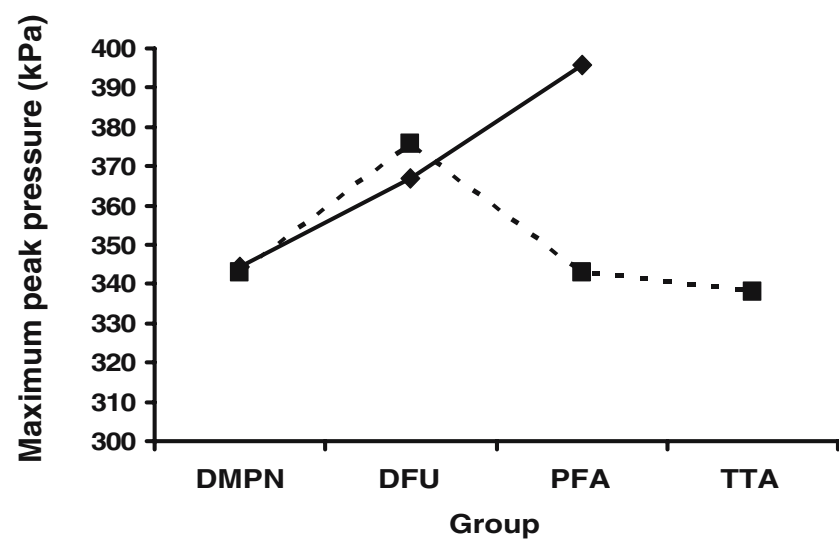

Fig. 4 The maximum peak pressure over the total surface area of the affected foot is demonstrated by the solid line and the contralateral foot is demonstrated by the broken line across the four diabetic groups. $D M P N$ Diabetic neuropathy, $D F U$ diabetic foot ulcer, $P F A$ partial foot amputation, TTA trans-tibial amputation not locate any direct evidence to support the linear relationship between the volume of walking activity and H-R QOL, further research in this area would be necessary to explore the precise relationship between the two variables, if any.

Although our findings confirm the reduction in daily walking activity and the need for improving the physical fitness of this patient population, the plantar pressure distribution on the affected and the contralateral foot during walking needs to be considered before walking can be recommended as a safe form of physical activity to improve fitness.

People with DMPN are known to present with a slower gait pattern [29]. Our results demonstrate a progressively gradual decline in the gait velocity as we proceed through the four groups (Fig. 3) indicating a progressive deterioration in the gait pattern. Gait velocity was used as a covariate in the analysis of peak plantar pressures because slower walking speed is known to coincide with decreased pressures over some areas of the foot [26]. On the affected limb, despite the gradual fall in the gait velocity, the results demonstrate a rise in the MPP values over the total foot from DMPN to PFA (Fig. 4). Higher peak pressures are already documented in patients with DFUs and PFAs on the affected side $[12,13]$. The increased plantar stress on the ulcerated foot and the reduction in the total contact area of the partially amputated foot might explain the higher average MPP values, indicating an increased risk of injury to the foot. Our findings did not demonstrate significantly increased MPP values over the fore-foot areas as reported in other studies. Although the majority of patients had forefoot ulceration, the averaging effect of various sites of ulcers probably masked the otherwise pronounced individual peak pressures. It should therefore be noted that the findings from this study indicate the potential risk of plantar injury to the entire foot, but the study is limited in providing information necessary to indicate risk to the specific plantar area.

On the contralateral side, the surviving foot of the patients with TTAs has received attention in the past with the objective of evaluating the risk of injury [11, 30]. The authors have demonstrated in a previous study that despite walking $30 \%$ more slowly than diabetic neuropathic subjects, patients with TTAs experienced increased plantar stress on the surviving foot during walking. Adaptations in gait and level of walking activity were identified to affect the plantar pressure distribution and ultimately the risk of ulceration to the surviving foot [30].

However, little is known about the pressure distribution over the contralateral foot among the patients with current DFUs and unilateral PFAs, despite the existing risk of plantar injury due to the presence of DMPN. Our results demonstrated a significant difference in the MPP on the heel 
and the medial metatarso-phalangeal regions of the contralateral foot across the four groups, demonstrating a significant rise in the heel peak pressures. Although the authors resisted multiple testing because of the danger of falsepositive results, it is interesting to note that the DFU group demonstrated an $8.7 \%$ rise in average total foot pressures and a $12.1 \%$ rise in average MPP over the medial MTs compared with the DMPN group, despite walking significantly more slowly. The protective mechanism adopted by the patients with DFUs to safeguard the ulcerated foot during walking, more specifically an increased push-off with the contralateral foot, might explain the compensatory increase in peak pressures on the contralateral foot.

Similarly, patients with PFAs demonstrated increased average MPP values on the heel of the contralateral foot compared with the controls. Adaptations in the gait pattern resulting from PFA can explain the increased plantar stress on the contralateral foot. Garbalosa et al. have reported a significantly greater maximum dynamic dorsiflexion range of motion during walking on the contralateral ankle compared with the ankle on the affected side following transmetatarsal amputation [31], which might result in a pronounced heel strike, explaining the greater plantar stress on the contralateral heel of the patients with PFAs in our study.

The TTA group presented with peak pressures comparable with the control group, despite a 39\% slower walking speed. Given the decreased capacity and performance of walking in this patient group, one might be tempted to increase the volume of walking activity. But the pressure picture suggests cautious implementation of rehabilitation programmes. Efforts to restore a near normal volume of ambulation in this patient population might increase the risk of plantar injury due to the rise in moderate repetitive stress, which is as equally instrumental as abnormally higher instantaneous stress in the development of plantar ulceration [32].

In summary, all the four groups in this study are already at risk of plantar injury due to the presence of DMPN [33]. Further complications, such as plantar ulceration, PFAs and TTAs, increase the risk of plantar injury in this patient population during walking. However, since walking is an integral component of activities of daily life [34], we suggest maintenance of essential daily walking in protective footwear with foot care $[35,36]$ and appropriate modifications in the gait pattern to reduce the MPP [37].

Concurrently the vital need to optimise physical fitness in this population could be addressed with partial weight-bearing or non-weight-bearing aerobic exercises as supplementary safe options. However, further research is needed to arrive at a specifically tailored aerobic exercise programme with equal emphasis on foot care and physical fitness for this patient population, which presents with specific needs at different stages of foot complications.

\section{Conclusion}

The diabetic foot is already at risk of plantar injury in the presence of neuropathy. With consequent stages of foot complications, the risk of injury increases further. Although the positive effects of walking on the cardio-respiratory system and glycaemic control are established, its impact on the neuropathic foot, leading to further complications, does not support its prescription as the solitary best option to improve physical fitness in the diabetic population. In order to optimise the functional outcome of walking without paying the price of increased risk of plantar injury to the diabetic foot, maintenance of essential walking in protective footwear along with appropriate modifications in the gait pattern and adequate foot care is recommended. Diabetic patients with plantar ulceration and PFAs need protection not only on the affected foot, but also on the contralateral foot to prevent the increased risk of plantar injury. Ambulation programmes among diabetic patients with TTA should, of necessity, be implemented with caution.

Concurrently, alternative forms of partial or non-weightbearing aerobic exercises could be considered supplementary to essential walking exercise to improve the level of physical fitness and glycaemic control of this patient population.

Acknowledgements We sincerely acknowledge the efforts of the staff of the Vascular Surgery, Podiatry Services and Artificial Limb Centres within the Cardiff \& Vale NHS Trust and Morriston Hospital Trust, Swansea, UK for their help in recruiting suitable subjects for the study. We are also grateful to the staff of the Research Centre for Clinical Kinaesiology, Cardiff University and the Wound Healing Research Unit for their assistance throughout the study and are grateful to all the participants in the study. The study was funded by the School of Health Care Studies, Cardiff University and the Wound Healing Research Unit.

R. W. M. van Deursen, the corresponding author for this paper, has full access to the data in the study and had the final responsibility for the decision to submit the paper for publication.

\section{References}

1. Yamanouchi K, Shinozaki T, Chikada K et al (1995) Daily walking combined with diet therapy is a useful means for obese NIDDM patients not only to reduce body weight but also to improve insulin sensitivity. Diabetes Care 18:775-778

2. Swartz AM, Strath SJ, Bassett DR Jr et al (2003) Increasing daily walking improves glucose tolerance in overweight women. Prev Med 37:356-362

3. Bhaskarabhatla KV, Birrer R (2004) Physical activity and type 2 diabetes. Tailoring exercise to optimize fitness and glycemic control. Phys Sportsmed 32:1-5

4. Reiber GE, Vileikyte L, Boyko EJ et al (1999) Causal pathways for incident lower-extremity ulcers in patients with diabetes from two settings. Diabetes Care 22:157-162

5. Reiber GE, Pecoraro RE, Koepsell TD (1992) Risk-factors for amputation in patients with diabetes mellitus - a case-control study. Ann Intern Med 117:97-105 
6. Lynch T, Kanat IO (1991) Transmetatarsal amputation-a literature review and case study. J Am Podiatr Med Assoc 81:540-544

7. Hosch J, Quiroga C, Bosma J, Peters EJG, Armstrong DG, Lavery LA (1997) Outcomes of transmetatarsal amputations in patients with diabetes mellitus. J Foot Ankle Surg 36:430-434

8. Armstrong DG, Lavery LA, Harkless LB, VanHoutum WH (1997) Amputation and reamputation of the diabetic foot. J Am Podiatr Med Assoc 87:255-259

9. Armstrong DG, van Schie CHM, Nguyen HC, Boulton AJM, Lavery LA, Harkless LB (2001) Off-loading the diabetic foot wound - a randomized clinical trial. Diabetes Care 24:1019-1022

10. Tudor-Locke CE, Bell RC, Myers AM, Harris SB, Lauzon N, Rodger NW (2002) Pedometer-determined ambulatory activity in individuals with type 2 diabetes. Diabetes Res Clin Pract 55:191-199

11. Veves A, Vanross ERE, Boulton AJM (1992) Foot pressure measurements in diabetic and nondiabetic amputees. Diabetes Care 15:905-907

12. Stess RM, Jensen SR, Mirmiran R (1997) The role of dynamic plantar pressures in diabetic foot ulcers. Diabetes Care 20:855-858

13. Armstrong DG, Lavery LA (1998) Plantar pressures are higher in diabetic patients following partial foot amputation. Ostomy Wound Manage 44:30-39

14. Towards a common language for functioning, disability and health ICF (2002) World Health Organization, Geneva

15. Kumar S, Fernando DJS, Veves A, Knowles EA, Young MJ, Boulton AJM (1991) Semmes-Weinstein monofilaments - a simple, effective and inexpensive screening device for identifying diabetic patients at risk of foot ulceration. Diabetes Res Clin Pract 13:63-67

16. Diamond JE, Mueller MJ, Delitto A, Sinacore DR (1989) Reliability of a diabetic foot evaluation. Phys Ther 69:797-802

17. Hood VL, Granat MH, Maxwell DJ, Hasler JP (2002) A new method of using heart rate to represent energy expenditure: the total heart beat index. Arch Phys Med Rehabil 83:1266-1273

18. Brooks D, Hunter JP, Parsons J, Livsey E, Quirt J, Devlin M (2002) Reliability of the two-minute walk test in individuals with transtibial amputation. Arch Phys Med Rehabil 83:1562-1565

19. Shepherd EF, Toloza E, McClung CD, Schmalzried TP (1999) Step activity monitor: increased accuracy in quantifying ambulatory activity. J Orthop Res 17:703-708

20. Hartsell H, Fitzpatrick D, Brand R, Frantz R, Saltzman C (2002) Accuracy of a custom-designed activity monitor: implications for diabetic foot ulcer healing. J Rehabil Res Dev 39:395-400

21. Mcpoil TG, Cornwall MW, Dupuis L, Cornwell M (1999) Variability of plantar pressure data - a comparison of the twostep and midgait methods. J Am Podiatr Med Assoc 89:495-501

22. Guy RJ, Clark CA, Malcolm PN, Watkins PJ (1985) Evaluation of thermal and vibration sensation in diabetic neuropathy. Diabetologia 28:131-137
23. Mueller MJ, Minor SD, Sahrmann SA, Schaaf JA, Strube MJ (1994) Differences in the gait characteristics of patients with diabetes and peripheral neuropathy compared with age-matched controls. Phys Ther 74:299-308

24. Burnfield JM, Few CD, Mohamed FS, Perry J (2004) The influence of walking speed and footwear on plantar pressures in older adults. Clin Biomech 19:78-84

25. Green SB, Salkind NJ (2003) One-way analysis of covariance In: Green SB, Salkind NJ (eds) Using SPSS for Windows and Macintosh: analyzing and understanding data. Prentice Hall, New Jersey, pp 191-201

26. Rosenbaum D, Hautmann S, Gold M, Claes L (1994) Effects of walking speed on plantar pressure patterns and hindfoot angular motion. Gait Posture 2:191-197

27. Wei M, Gibbons LW, Kampert JB, Nichaman MZ, Blair SN (2000) Low cardiorespiratory fitness and physical inactivity as predictors of mortality in men with type 2 diabetes. Ann Intern Med 132:605-611

28. Tennvall GR, Apelqvist J (2000) Health-related quality of life in patients with diabetes mellitus and foot ulcers. J Diabetes Complications 14:235-241

29. Menz HB, Lord SR, St George R, Fitzpatrick RC (2004) Walking stability and sensorimotor function in older people with diabetic peripheral neuropathy. Arch Phys Med Rehabil 85:245-252

30. Kanade RV, van Deursen RWM, Price P, Harding K (2006) Risk of plantar ulceration in diabetic patients with single-leg amputation. Clin Biomech 21:306-313

31. Garbalosa JC, Cavanagh PR, Wu G et al (1996) Foot function in diabetic patients after partial amputation. Foot Ankle Int 17:43-48

32. Brand PW (1991) The insensitive foot (including leprosy). In: Jahss MH (ed.) Disorders of the foot and ankle. Medical and surgical management. WB Saunders, Philadelphia, Pennsylvania, pp 2170-2186

33. Frykberg RG, Harvey C, Lavery LA, Harkless L, Pham H, Veves A (1998) Role of neuropathy and high foot pressures in diabetic foot ulceration. Diabetes Care 21:1714-1719

34. Bates JH, Serdula MK, Khan LK, Jones DA, Gillespie C, Ainsworth BE (2005) Total and leisure-time walking among US adults - should every step count? Am J Prev Med 29:46-50

35. Cavanagh PR (2004) Therapeutic footwear for people with diabetes. Diabetes Metab Res Rev 20:S51-S55

36. Hunt D (2002) Using evidence in practice-foot care in diabetes. Endocrinol Metab Clin North Am 31:603-611

37. Brown HE, Mueller MJ (1998) A 'step-to' gait decreases pressures on the forefoot. J Orthop Sports Phys Ther 28:139-145

38. Busse ME (2004) Co-activation, muscle weakness and functional ability in patients with different neurological conditions. Cardiff University, Cardiff 